PROCEEDINGS OF THE

AMERICAN MATHEMATICAL SOCIETY

Volume 125, Number 1, January 1997, Pages 75-81

S 0002-9939(97)03859-8

\title{
SMALL INFINITE DIMENSIONAL MODULES FOR ALGEBRAIC GROUPS
}

\author{
ANDY R. MAGID
}

(Communicated by Eric M. Friedlander)

\begin{abstract}
A infinite dimensional module for an algebraic group is called small provided every proper submodule is finite dimensional. Small infinite dimensional modules exist provided that the characteristic is zero and the group has a non-trivial unipotent radical. The unipotent radical is shown to act through an abelian quotient, which allows a description, up to finite dimensional quotients, of the SID modules with trivial module socle via equivariant commutative algebra. In the case that the group is in fact unipotent, this description is used to calculate the Hilbert function of the ascending socle series of the module.
\end{abstract}

\section{INTRODUCTION}

Let $G$ denote an algebraic group over the algebraically closed field $k$ of characteristic zero. As Donkin has shown [D], a rational $G$-module $M$ satisfies the descending chain condition (DCC) provided the socle of $M$ is finite dimensional (and conversely, of course).

One of the equivalent forms of the descending chain condition on a module is that any class of submodules contains minimal elements, namely submodules all of whose proper submodules lie outside the class. These minimal modules may be thought of as being on the edge of the class, and as embodying the minimum necessary for entry into the class. As such, the analysis and classification of their structure is of interest.

Any infinite dimensional $G$-module with DCC contains minimal such, namely infinite dimensional $G$-modules all of whose proper submodules are finite dimensional. We call a module with this latter property small and abbreviate small infinite dimensional by SID. (Others use the terminology 'just infinite'.) Clearly SID modules also have DCC.

When we classify SID modules, in addition to isomorphism we will also use the notion of virtual isomorphism: SID modules are said to be virtually isomorphic if they have isomorphic non-zero quotients (which means, of course, that they are isomorphic modulo finite dimensional submodules). It is clear that virtual isomorphism is an equivalence relation.

As an example of an SID module, we can take $G=\mathbb{G}_{a}$ (the additive group) and $M=k[G]$ (its coordinate ring): using the identity $t: G \rightarrow k$ as the coordinate,

Received by the editors July 31, 1995.

1991 Mathematics Subject Classification. Primary 20G99.

Partially supported by NSA grant MDA904-95-H-1038. 
we have $k[G]=k[t]$ (polynomials) with action $g \cdot t=g+t$. Then any submodule containing a polynomial of degree $n$ must also contain $1, t, t^{2}, \ldots, t^{n}$, from which it follows that the only proper submodules are $E_{i}=k 1+k t+\cdots+k t^{i}, i=0,1, \ldots$. (Note that characteristic zero is being used strongly here, and that in characteristic $p>0 k\left[\mathbb{G}_{a}\right]=k[t]$ contains the infinite descending chain of submodules $k[t] \supset$ $\left.k\left[t^{p}\right] \supset k\left[t^{p^{2}}\right] \supset \ldots\right)$

If $G$ is reductive then every module is its own socle, so it has no SID modules. Otherwise, $G$ has a non-trivial unipotent radical $U$ and can be written as a semidirect product $G=H U$ where $H$ is a maximal reductive subgroup. Let $E_{G}(k)$ denote the injective hull of the trivial $G$-module $k$; we have $E_{G}(k)=k[U]$, where $U$ acts by translation and $H$ by conjugation: $p u \cdot f(v)=f\left(p^{-1} v p u\right)$ for $p \in H$ and $u, v \in U$. In particular, it is infinite dimensional. The socle of $E_{G}(k)$ is just $k$, and hence it has DCC. In particular, it contains an SID submodule. Thus we conclude that a group has an SID module exactly when its unipotent radical is non-trivial. So the appropriate place to begin the study of SID modules is for unipotent groups.

\section{UNIPOTENT GROUPS}

Definition 1. Let $U$ be a unipotent algebraic group and let $E$ be a $U$ module. Define submodules of $E$ by $E_{0}=\{0\}$ and if $E_{i}$ is defined then $E_{i} \subseteq E_{i+1}$ is defined by

$$
\left(E / E_{i}\right)^{U}=E_{i+1} / E_{i} .
$$

We have $E_{0} \subset E_{1} \subset \ldots$ with $\cup E_{i}=E$.

By the definition $x \in E_{i+1}$ if and only $(g-1) x \in E_{i}$ for all $g \in U$. Then an easy induction establishes that

$$
E_{i}=\left\{x \in E \mid\left(g_{1}-1\right) \ldots\left(g_{i}-1\right) x=0 \quad \forall \quad g_{1}, \ldots, g_{i} \in U\right\} .
$$

Note that (i) implies that if $x \in E_{j}$ and $g_{1}, \ldots, g_{k} \in U$ then $\left(g_{1}-1\right) \ldots\left(g_{k}-1\right) x \in$ $E_{j-k}$.

It's obvious from the above formula (i) (or even from the definition) that $(\cdot)_{i}$ is a functor on $U$ modules which commutes with direct sums and carries monomorphisms to monomorphisms.

If $E=k[U]$, then each $E_{i}$ is finite dimensional [LM1, 1.3.2, p.326]. Moreover $k[U]$ is also the injective hull $E_{U}(k)$ of the trivial $U$ module $k$ [CPS, p.4]. If $M$ is a $U$ module with $n$-dimensional socle $M^{U}$, then its injective hull is the injective hull of its socle, and the latter is $k[U]^{(n)}$. It follows that every $M_{i}$ is also finite dimensional. In particular, each $M / M_{i}$ also has finite dimensional socle.

In the case that $E_{1}$ (and hence by the above all $E_{i}$ ) is finite dimensional, we can define a Hilbert function for $E$ :

Definition. Let $E$ be a module for the unipotent group $U$ with finite dimensional socle. The function $f(E, i)=\operatorname{dim}_{k}\left(E_{i}\right)$ is called the Hilbert function of $E$.

If $M$ is any $U$ module and $g \in U$ then $m \mapsto g \cdot m-m$ defines a $k$-linear endomorphism of $M$ which we denote $g-1$ as above. For $a_{0}, \ldots, a_{m} \in k$ we then have a polynomial endomorphism $\sum a_{i}(g-1)^{i}$. Since formula (i) implies that $g-1$ is locally nilpotent, we can even define a power series endomorphism $\sum a_{i}(g-1)^{i}$ for an infinite sequence of elements $a_{i}, i=0,1,2, \ldots$, of $k$. In case $g$ is in the center $Z(U)$ of $U$, these $k$-linear endomorphisms are $U$ linear.

In the SID case, we have the following fact about $U$ endomorphisms: 
Proposition 1. Let $E$ and $F$ be SID $U$ modules and let $T \in H_{0}(E, F)$. Then either $T=0$ or $T$ is onto.

If $E=F$ then $T \in \operatorname{End}_{U}(E)$ is a non-unit if and only if $\operatorname{Ker}(T) \neq 0$, and if $E^{U}=k$, then $\operatorname{End}_{U}(E)$ is local.

Proof. If $T \neq 0$ then the kernel $K$ of $T$ is a proper, hence finite dimensional, submodule of $E$. If $T$ is not onto, then the image $I$ of $T$ is a proper, hence finite dimensional, submodule of $F$. So if $T$ were neither 0 nor onto the exact sequence

$$
0 \rightarrow K \rightarrow E \rightarrow I \rightarrow 0
$$

would imply that $E$ is finite dimensional, contrary to hypothesis. It follows immediately that if $E=F$ then the invertible endomorphisms are exactly the monomorphisms. If $E^{U}=k$, then $\operatorname{Ker}(T)^{U}$ is either $k$ or 0 , with the latter occurring only when $\operatorname{Ker}(T)=0$. Thus $T$ is a non-unit exactly when $T(1)=0$, from which it follows that the non-units of $\operatorname{End}_{U}(E)$ form an ideal.

We want to apply the proposition to the case of an endomorphism on an SID module induced by multiplication by a group element:

Proposition 2. Let $E$ be an SID $U$-module. Let $u \in Z(U)$ be central in $U$ with $E^{\langle u\rangle}$ finite dimensional. Let $T=u-1 \in \operatorname{End}_{U}(E)$ and define $\phi: k[[t]] \rightarrow \operatorname{End}_{U}(E)$ by $t \mapsto T$. Consider $E$ as a $k[[t]]$ module via $\phi$. Then $E$ is a faithful, torsion, divisible $k[[t]]$ module with finite dimensional socle, and $\operatorname{End}_{k[[t]]}(E)$ is a matrix algebra over $k[[t]]$ of size equal to this finite dimension.

Proof. Note that $\phi$ is defined since for $x \in E$ there is some $k$ with $t^{k} x=0$. Let $R=k[[t]]$ and let $I \subset R$ be the kernel of $\phi$. As an ideal of $R$, if $I$ is non-zero it must be generated by a power of $t$, say $t^{k}$. But then $T^{k}=(u-1)^{k}$ is zero on $E$. On the other hand, by assumption $T \neq 0$, and thus by Proposition $1 T$ is onto. So $I$ is zero and $E$ is a faithful $R$ module. If $x \in E$ then $x \in E_{i}$ for some $i$, so that $t^{i} x=0$ by formula (i). Thus $E$ is a torsion $R$ module. Since by Proposition 1 non-zero elements of $R$ give surjective endomorphisms of $E, E$ is a divisible $R$ module. Since the divisible $R$ modules are precisely the injective ones, any injective $R$ module is a direct sum of copies of indecomposable injectives, and the indecomposables are either the fraction field $k((t))$ or the injective hull of the residue field $E_{R}(k)$, and only the latter has torsion, we see that $E$ is a direct sum of copies of $E_{R}(k)$ as an $R$ module. The number of copies is the dimension of $\{x \in E \mid t x=0\}$, that is, of the kernel of $T$, which, being a proper $U$ submodule, is finite dimensional, say of dimension $n$. Thus $E$ is isomorphic to $E_{R}(k)^{(n)} ;$ since $\operatorname{End}_{R}\left(E_{R}(k)\right)=R$, we then have that $\operatorname{End}_{R}(E)=M_{n}(R)$ as desired.

We retain the hypotheses and notation of Proposition 2 ( $E$ is an SID $U$-module, $u \in Z(U)$ acts non-trivially on $E$ with finite dimensional fixed point set, and $E$ is regarded as a $k[[t]]$ module via $t \mapsto u-1)$. Now suppose $g$ is any element of $U$ and consider the $k$-linear automorphism $\rho(g)$ of $E$ given by $x \mapsto g x$. Since $u$ is central, $\rho(g)$ commutes with $u-1$ and hence is $k[[t]]$ linear. Thus $\rho$ is actually a representation of $U$ over $k[[t]]$ :

$$
\rho: U \rightarrow G L_{n}(k[[t]]) .
$$

Moreover, $\rho(u)$ is the scalar matrix $(1+t) I_{n}$. As we now see, the existence of such a representation has strong implications for the structure of $E$ as a $U$-module. 
Theorem 3. Let $E$ be a small infinite dimensional module for the unipotent group $U$. Then the commutator subgroup of $U$ acts trivially on $E$.

Proof. We replace $U$ by the kernel of the representation on $E$ so that we may assume that $E$ is a faithful $U$ module. Then we want to show that $U$ is abelian. If not, then there exist $g, h, u \in U$ such that $u \in Z(U)$ is central and we have the commutator equation $(g, h)=u$. Let $\Gamma$ be the (abstract) subgroup of $U$ generated by $g, h, u$. As above, $\Gamma$ is faithfully represented in $G L_{n}(k[[t]])$, and hence in $G L_{n}(K)$, where $K$ is the algebraic closure of $k((t))$; we regard this representation as an inclusion. Let $\bar{\Gamma}$ be the Zariski closure of $\Gamma$. Since $\Gamma$ is nilpotent, so is its Zariski closure, and we have $\bar{\Gamma}=N \times T$ where $N$ is a unipotent algebraic group (over $K$ ) and $T$ is a diagonalizable group whose connected component $T^{0}$ is the unique maximal torus of $\bar{\Gamma}$. Recall that $u=(1+t) I_{n}$ is scalar and hence contained in the one dimensional torus $(K-\{0\}) I_{n}$. It follows that the Zariski closure of the cyclic subgroup of $\Gamma$ generated by $u$ is this torus, and hence that the scalar torus is contained in $T^{0}$ and hence in $T$. Let $p r: \bar{\Gamma}=N \times T \rightarrow T$ be the projection. We have just seen that $\operatorname{pr}(u)=(1+t) I_{n}$, and hence $\operatorname{pr}\left(u^{m}\right) \neq 1$ for any $m>0$. On the other hand, since $\left[T: T^{0}\right]<\infty$ there is an $n$ such that $\operatorname{pr}\left(g^{n}\right) \in T^{0}$ and $\operatorname{pr}\left(h^{n}\right) \in T^{0}$. But $\left(g^{n}, h^{n}\right)=u^{m}$ (for appropriate $m$ ) and applying $p r$ then gives the impossible equation $1 \neq \operatorname{pr}(u)^{m}=\left(\operatorname{pr}(g)^{n}, \operatorname{pr}(h)^{n}\right)$ in the abelian group $T^{0}$. We conclude that $U$ must be abelian.

\section{Commutative unipotent CAse}

Theorem 3 reduces the study of SID modules for unipotent groups to commutative algebra. We first recall the construction of the complete group algebra $k\langle\langle U\rangle\rangle$ [LM2, p.536]: as a ring, this is just the $U$ module endomorphism ring of the injective hull $E_{U}(k)$ of the trivial $U$ module. Since $E_{U}(k)=k[U]$ as a left $U$-module, and $U$ also acts (U- equivariantly) on the right on $k[U]$, there is an embedding of $U$ into $\operatorname{End}_{U}\left(E_{U}(k)\right)=k\langle\langle U\rangle\rangle$. Thus modules for the complete group algebra are by restriction (abstract) $U$-modules. There is an augmentation $k\langle\langle U\rangle\rangle \rightarrow k$ by $T \mapsto T(1)$ whose kernel is denoted $J_{1}(U)$. The discrete $k\langle\langle U\rangle\rangle$-modules are those for which every element is annihilated by a power of $J_{1}(U)$. The $U$ module obtained by restriction from a discrete $k\langle\langle U\rangle\rangle$ module is rational, and every rational $U$-module arises in this way [LM2, 2.8, p.539]. The complete group algebra is isomorphic as a topological ring to the completion of the universal enveloping algebra of the Lie algebra of $U$ at its augmentation ideal, and under this isomorphism $J_{1}$ is the ideal of the completion generated by that augmentation ideal [LM2, 1.8, p.535].

In particular, if $U$ is commutative then $k\langle\langle U\rangle\rangle$ is a (commutative) formal power series algebra $R=k\left[\left[t_{1}, \ldots, t_{n}\right]\right](n=\operatorname{dim}(U)), J_{1}(U)=\left(t_{1}, \ldots, t_{n}\right)=M$ is the maximal ideal, and $U$-modules are $R$-modules every element of which is annihilated by a power of $M$ ( $M$-primary modules). Moreover, if $X$ is such a module, then the $U$ - submodule $X_{i}$ is $\operatorname{Hom}_{R}\left(R / M^{i}, X\right)$. Such a module will be SID if it is infinite dimensional but every proper submodule is finite dimensional.

We can use the $R$-module injective hull $E_{R}(R / M)$ of the simple $R$-module to create the standard contravariant duality between $M$ primary modules and complete modules $X^{D}=\operatorname{Hom}_{R}\left(X, E_{R}(R / M)\right)$ which is exact since $E_{R}(R / M)$ is injective and perfect since $E_{R}(R / M)^{D}=R$ and $R^{D}=E_{R}(R / M)$. Under the duality, we have (for an $M$-primary module) that $X_{i}^{D}=X / M^{i} X$. If $E$ is an SID $R$ module, 
then $E^{D}$ is a finitely generated $R$-module all of whose proper quotients are finite dimensional, and conversely. It remains to study such $R$-modules.

Such modules $X$ are finitely generated over $R$, infinite dimensional over $k$, but all their proper quotients are $k$ finite dimensional. We will call these Dual Infinite Small $R$-modules, or $D I S$ modules. Any non-zero submodule $Y \subseteq X$ has these same properties and $Y$ is of finite codimension in $X$. In particular, if $P$ is an associated prime of $X$ then $R / P$ is DIS. But this can only happen if $P$ is of codimension one (or zero). Moreover, it follows easily from the fact that $X$ is DIS that there is at exactly one codimension one associated prime of $X$.

Conversely, if $X$ is a finitely generated $R$-module (infinite dimensional over $k$ ), with $\operatorname{Ass}(X) \subseteq\{P, M\}$ where $P$ is codimension one and $R / P$ is of finite codimension in $X$ then $X$ is DIS.

Finally, we conclude that if $X$ is a DIS module with associated codimension one prime $P$ then there is a finite dimensional submodule $N$ of $X^{D}$ with $X^{D} / N$ isomorphic to $(R / P)^{D}$.

It is clear from the above discussion that the classification of SID modules for the commutative group $U$ (which is equivalent to the classification of DIS modules for the ring $R$ ) is at least as hard as the classification of codimension one prime ideals of $R$. On the other hand, this is the only difficulty: we can virtually classify SID modules up to the classification of codimension one primes:

Theorem 4. Let $U$ be a unipotent algebraic group, with $U^{\mathrm{ab}}$ of dimension $d$ over $k$. Then there is a bijection between the set of virtual isomorphism classes of SID modules and the set of codimension one prime ideals of $k\left[\left[t_{1}, \ldots, t_{d}\right]\right]$.

Proof. By Theorem 3, SID $U$ modules are (SID) $U^{\text {ab }}$-modules and vice versa. Thus we may assume $U$ is abelian and that $R=k\langle\langle U\rangle\rangle=k\left[\left[t_{1}, \ldots, t_{n}\right]\right]$ as above. Let $E$ be an SID $U$ - module. As noted in the above discussion, the DIS $R$-module $E^{D}$ has a unique associated codimension one prime $P$, and the submodule $R / P \subseteq E^{D}$ is of finite codimension. Then, applying $D$, we have a surjection $E=E^{D D} \rightarrow(R / P)^{D}$ so that $E$ is virtually isomorphic to $(R / P)^{D}$. Suppose $Q$ is also a codimension one prime ideal and that $(R / P)^{D}$ and $(R / Q)^{D}$ are virtually isomorphic, with $F$ denoting their (SID) common quotient. Then both $R / P$ and $R / Q$ are submodules of $F^{D}$. Since $F^{D}$ has a unique associated prime of codimension one, we conclude that $Q=P$. If $E^{\prime}$ is an SID module virtually isomorphic to $E$, then as above $E^{\prime}$ has a quotient $\left(R / P^{\prime}\right)^{D}$ where $P^{\prime}$ is codimension one, and since $(R / P)^{D}$ and $\left(R / P^{\prime}\right)^{D}$ are virtually isomorphic, $P=P^{\prime}$. Thus $P$ is an invariant of the virtual isomorphism class of $E$, and the theorem follows.

The proof of Theorem 4 also provides a calculation of Hilbert functions, as we now see:

Proposition 5. Let $E$ be an SID module for the unipotent group $U$. Then the Hilbert function of $E$ is a linear polynomial in $i$, for $i$ sufficiently large.

Proof. We may replace $U$ by its image on $E$ and assume that $U$ is abelian. Next, note that if $F$ is a finite dimensional submodule of $E$ then $f(E, i)=f(E / F, i)+$ $\operatorname{dim}(F)$ for large $i$. So we can replace $E$ by $E / F$ as needed. In the notation of the proof of Theorem 4, we can take $E / F=(R / P)^{D}$ where $P$ is a codimension one prime ideal of $R$. Since, in the notation of the discussion preceding Theorem 4, $\left((R / P)^{D}\right)_{i}=\left((E / F)_{i}\right)^{D}$ we have $\operatorname{dim}_{k}\left((E / F)_{i}\right)=\operatorname{dim}_{k}\left((R / P) /\left(M^{i}(R / P)\right)\right)$, the 
Hilbert function of $E / F$ and $R / P$ are the same. Since the Krull dimension of $R / P$ is one, its Hilbert function is linear for large $i$, and hence so is $f(E / F, i)$.

\section{General Groups}

We now turn to the case of a general algebraic group $G$ with unipotent radical $U=R_{u}(G)$ and a maximal reductive subgroup $H$. We assume that $U \neq\{e\}$ so that $G$ has SID modules. Our first result implies that we may take $U$ abelian:

Proposition 6. Let $E$ be an SID module for the algebraic group $G$. Then $E$ has a finite dimensional $R_{u}(G)$ socle and $\left(R_{u}(G), R_{u}(G)\right)$ acts trivially on $E$.

Proof. Let $U=R_{u}(G)$. The $G$ socle of $E$ coincides with $E^{U}$ and hence $E$ has finite dimensional socle as $U$ module also. Hence we can select an SID $U$-submodule $E^{\prime} \subseteq E$. By Proposition, $(U, U)$ acts trivally on $E^{\prime}$. If $u \in(U, U), g \in G$, and $x \in E^{\prime}$, then $u g x=g\left(g^{-1} u g\right) x=g x$. It follows that $(U, U)$ acts trivially on $\sum_{g \in G} g E^{\prime}$; but the latter is an infinite dimensional $G$-submodule of $E$ so must be $E$ itself.

We continue to use the notation $G=U H$ and recall again that the injective hull $E_{G}(k)$ of the trivial $G$-module is isomorphic to $k[U]$ with $U$ acting as left translations and $H$ on the right through conjugation. We assume further that $U$ is abelian and let $R=k\langle\langle U\rangle\rangle$ which is as before a power series algebra, on which $H$ acts by conjugation. (Since $U$ is abelian, we could equally well consider the conjugation action of $G$.) $H$ acts on $R$ by algebra automorphisms and preserves the maximal ideal $M$ of $R$. We note above that $k[U]$ is the injective hull of $k$ both over $U$ and $R$, and with the action of $H$ also the injective hull over $G$. Here we want to regard it as the injective hull $E_{R}(k)$ over $R$ but also with compatible $H$ action.

Now let $X$ be any $G$ module with finite dimensional socle. Since as a $U$ module it also has finite dimensional socle, we have that $X$ is also an ( $M$ - primary) $R$ module. $H$ acts both on $X$ and $R$ and the actions are compatible. Extending the usual terminology, we will call such an $X$ an $R \cdot H$-module. The action of $H$ on $X$ is rational (hence semi-simple); this is not true of the action on $R$ but is true of the induced action on its associated graded ring $\oplus_{i \geq 0} M^{i} / M^{i+1}$.

Suppose that $V$ is the finite dimensional socle of the $G$ module $X$. Then the injective hulls of $X$ and $V$ coincide, and the latter can be identified with $E_{R}(k) \otimes V$, and we have an essential monomorphism $X \rightarrow E_{R}(k) \otimes V$.

The actions of $H$ on $X$ and $E_{R}(k)$ make $X^{D}=\operatorname{Hom}_{R}\left(X, E_{R}(k)\right)$ also an $R \cdot H$ module with $X \rightarrow X^{D D}$ an $R \cdot H$ isomorphism; we thus get a duality between $H$-semisimple $R \cdot H$ modules with finite dimensional socle and certain $R$ finitely generated $R \cdot H$ modules: if $X$ has socle $V$ so that $X$ embeds in $E_{R}(k) \otimes V$, then there is a surjection $E_{R}(k)^{D} \otimes V \rightarrow X^{D}$, and $E_{R}(k)^{D}=R$. Under this duality, SID $G$ modules correspond to modules with all non-zero submodules of finite codimension.

In the general case, the classification of these dual modules will be quite complicated. But we can be more concise in case the SID $G$ module $X$ has trivial socle (that is, the socle is a trivial $G$ module). In this case we have an $R \cdot H$ surjection $R^{(n)} \rightarrow X^{D}$, where $n$ is the dimension of the socle of $X$.

For an SID module $X$, the action of $H$ on $X^{D}$ permutes the (finitely many) associated primes. If we assume further that $H$ is connected (so has no proper 
finite quotients), then this action must be trivial, and any associated prime $Q \subset R$ of $X^{D}$ is $H$-stable.

With the above two assumptions, we obtain the following generalization of Theorem :

Theorem 7. Let $X$ be an SID module for the connected algebraic group $G=U H$ and assume that the socle of $X$ is a trivial module. Suppose $U^{\mathrm{ab}}$ has dimension $d$. Then there is a unique $H$-stable prime ideal $Q$ of $R=k\langle\langle U\rangle\rangle=k\left[\left[t_{1}, \ldots, t_{d}\right]\right]$ such that $X$ is virtually isomorphic to $(R / Q)^{D}$. There are no $H$ stable primes of $R$ properly between $Q$ and $M=\sum R t_{i}$, and if $Q^{\prime}$ is any such prime ideal of $R$ then $\left(R / Q^{\prime}\right)^{D}$ is an SID $G$ module with trivial socle.

Proof. By Proposition 6, we can assume that $(U, U)$ acts trivially on $X$, and thus replace $G$ by $G /(U, U)$ and assume $U$ is abelian. As noted above, the triviality of the socle of $X$ implies an $R \cdot H$ surjection $R^{(n)} \rightarrow X^{D}$. Composing with the various coordinate inclusions we conclude that there is an $R \cdot H$ morphism $R \rightarrow X^{D}$ with cofinite dimensional image (isomorphic to) $R / I$. Every non-zero quotient of $R / I$ is finite dimensional. The minimal primes of $I$ are permuted by $H$ and hence $H$ stable by connectivity. If $M$ is the only minimal prime over $I$ then $R / I$ (and hence $X^{D}$ ) would be finite dimensional, contrary to hypothesis. Thus there is a prime $Q \supseteq I$ with $Q \neq M$ and $Q H$ stable. If $Q \neq I$ then $R / Q$ is an infinite dimensional proper quotient of $R / I$, and hence $Q=I$. The injection $R / Q \rightarrow X^{D}$ dualizes to the surjection and virtual isomorphism $X \rightarrow(R / Q)^{D}$. Because every proper quotient of $R / Q$ must be finite dimensional, we conclude that there are no prime ideals between $Q$ and $M$. If $X$ were virtually isomorphic to $(R / P)^{D}$ for some other $H$ stable prime $P$, then $R / P$ and $R / Q$ would have isomorphic cofinite dimensional $R$ submodules, which is impossible.

Finally, suppose $Q^{\prime}$ is an $H$ stable prime ideal of $R$ with no $H$ stable primes between it and $M$. A non-zero $R \cdot H$ submodule $Y$ of $R / Q^{\prime}$ corresponds to an $H$ stable ideal $I$ properly containing $Q$. Since by connectivity of $H$ the minimal primes over $I$ are $H$ stable and since they all properly contain $Q^{\prime}$ we have that $M$ is the unique minimal prime over $I$ and hence that $I$ is $M$ primary so that $R / I$ is finite dimensional. But then $Y$ is cofinite dimensional in $R / Q^{\prime}$. It follows that $\left(R / Q^{\prime}\right)^{D}$ is SID. Moreover, the $R \cdot H$ surjection $R \rightarrow R / Q^{\prime}$ dualizes to a $G$ injection $\left(R / Q^{\prime}\right)^{D} \rightarrow E_{R}(k)=E_{G}(k)$ so that the socle of $\left(R / Q^{\prime}\right)^{D}$ is seen to be $G$ trivial (and in fact be equal to $k$ ).

\section{REFERENCES}

[CPS] E. Cline, B. Parshall. and L. Scott, Induced modules and affine quotients, Math. Ann. 230 (1977), 1-14. MR 57:9861

[D] S. Donkin, On the Noetherian property of endomorphism rings of certain comodules, J. of Algebra 70 (1981), 394-419. MR 84e:16020b

[LM1] A. Lubotzky and A. Magid, Free prounipotent groups, J. of Algebra 80 (1983), 323-349. MR 85b: 14062

[LM2] Cohomology, Poincaré series, and group algebras of unipotent groups, Amer. J. Math. 107 (1985), 531-553. MR 86h:20063

Department of Mathematics, University of Oklahoma, Norman, Oklahoma 73019

E-mail address: amagid@uoknor.edu 